\title{
The Patient Educator Presentation in Dental Education: Reinforcing the Importance of Learning About Rare Conditions
}

\author{
Paul C. Edwards, MS, DDS; Jasmine Graham, BS; Rebecca Oling, MA, MLS; \\ Kate E. Frantz, MPH
}

Abstract: The aim of this study was to determine whether a patient educator presentation (PEP) on pemphigus vulgaris would increase second-year dental students' awareness of the importance of learning about rare conditions and improve their retention of rare disease knowledge. The study involved students' subjective assessments of a PEP experience at two U.S. dental schools. In this mixed methods study, cross-sectional data were obtained by surveys and in-depth interviews. Questions focused on students' assessment of the messages acquired from the PEP and its likely impact on their future clinical care. At University 1, students completed paper surveys with open-ended questions and participated in a focus group. At University 2, students completed an online survey consisting of rating scale and open-ended questions. Responses to open-ended questions were categorized into themes. At University 1, 79 students (out of a possible 102; response rate 77.5\%) completed the survey, and an additional ten students participated in a focus group. At University 2, 30 students (out of a possible 104; response rate 28.8\%) completed the survey. At Universities 1 and 2, 88\% and 100\%, respectively, of respondents stated the PEP would influence their future clinical decision making. The vast majority of respondents (94\% and $100 \%$ at University 1 and University 2, respectively) were of the opinion that the personal testimonial from a patient would help them recall information about pemphigus vulgaris in five years' time. Respondents from both universities commented that the PEP emphasized the importance of not dismissing a patient's concerns. These results suggest that a presentation by a patient with a rare condition can be an effective educational tool for preclinical dental students.

Dr. Edwards is Professor, Department of Oral Pathology, Medicine, and Radiology, Indiana University School of Dentistry; Ms. Graham is Content and Curriculum Intern, International Pemphigus \& Pemphigoid Foundation, Sacramento, CA; Ms. Oling is Coordinator of Instruction and Literature Librarian, Purchase College, State University of New York; and Ms. Frantz is Awareness Program Manager, International Pemphigus \& Pemphigoid Foundation. Direct correspondence to Dr. Paul C. Edwards, Department of Oral Pathology, Medicine, and Radiology, Indiana University School of Dentistry, 1121 West Michigan St., Room S104, Indianapolis, IN 46202-5186; 317-274-5136; pcedward@iu.edu.

Keywords: dental education, oral pathology, rare diseases, orphan diseases, pemphigus, patient educator

Submitted for publication 6/2/15; accepted 10/22/15

$I^{n}$ $\mathrm{n}$ the traditional model of dental education, the first year and a half to two years of the DDS curriculum typically focus on didactic biomedical science and preclinical lectures, supplemented with preclinical laboratory exercises. In many dental schools, the curriculum allows for only minimal direct patient contact during this period, so it can be a challenge for students to understand the importance of the many rare conditions covered in the didactic curriculum and their relevance to future dental practice. ${ }^{1}$ As a result, students often end up viewing significant portions of this aspect of their education as a hurdle that must be surmounted prior to engaging in patient care. ${ }^{2}$ In recognition of this concern, some dental schools have restructured their curricula to reinforce the relationship between the biomedical science curriculum and clinical care, by both introducing earlier clinical contact and extending the delivery of basic science content into the clinical phase of the curriculum.
However, even in the presence of these progressive curricular changes, it can remain a challenge to gain student understanding of the importance of this information in the context of overall patient care, especially considering the vast quantity of information to which dental students are exposed during their education. This perceived lack of clinical relevance can be particularly pronounced with respect to those conditions classified as "rare diseases," defined by the National Institutes of Health's Office of Rare Diseases Research as diseases affecting fewer than 200,000 people in the United States. ${ }^{3}$ Also referred to as "orphan diseases," by definition this group encompasses more than 6,800 individual conditions, a significant number of which present with head and neck manifestations. When one considers that together these rare diseases affect an estimated 25-30 million individuals in the United States, it is evident that dental practitioners will encounter a significant number of patients with one or more of 
these conditions in their day-to-day clinical practice. It is therefore critically important to impress upon dental students the importance of not losing track of the fact that although these conditions occur only rarely, as future health care providers they will be at the forefront in recognizing and managing these diseases in their patients. ${ }^{4}$

Dental students appear to have a strong visual learning preference. ${ }^{5}$ Therefore, we hypothesized that they would be more likely to appreciate the importance of the rare conditions presented as part of their introductory oral pathology course if this didactic information was supplemented with a live presentation by a patient with one of these conditions. Specifically, we hypothesized that a classroom presentation by a "patient educator" with a rare medicaldental condition would, through an emotional appeal, increase students' understanding of the importance of learning about rare conditions that can present with oral manifestations and thereby improve their rare disease knowledge retention. As this approach would require repurposing existing curricular time, its effectiveness needed to be assessed before it was recommended as an educational methodology.

The aim of this study was to determine whether a patient educator presentation (PEP) on pemphigus vulgaris would increase dental students' awareness of the importance of learning about rare conditions and their rare disease knowledge retention. We report on students' subjective assessments of a PEP, defined as a presentation by a real patient on his or her own illness, as a means of reinforcing the importance of understanding and recognizing less common conditions that may present with oral and maxillofacial features. The study was designed to compare findings from second-year dental students at two Midwestern dental schools.

\section{Materials and Methods}

The Indiana University Institutional Review Board determined that the study was exempt (Protocol 1408895631). The mixed methods study, comprising both quantitative and qualitative analysis, ${ }^{6}$ included cross-sectional data collection via paper and online surveys, supplemented with in-depth interviews.

In the intervention, a practicing nurse recently diagnosed with pemphigus vulgaris gave similar 45-minute talks, followed by ten-minute question and answer sessions, at University 1, Indiana Uni- versity, and University 2, the University of Michigan. Both presentations were delivered during regularly scheduled class sessions as components of mandatory second-year dental student courses in introductory oral and maxillofacial pathology.

The patient educator described her 18-month journey through the health care system prior to obtaining a definitive diagnosis for her condition, how she had initially presented to her primary care dentist with painful oral erosions, and how, following numerous unsuccessful attempts to obtain a diagnosis for her oral condition, she subsequently developed involvement of her rectal mucosa. The patient educator articulated her frustration at being treated empirically (including being advised to "brush better," prescribed mouthwash for her oral lesions, and treated with nitroglycerin cream and botulin toxin for an "anal fissure") without a definitive diagnosis by her general dentist, family physician, and colorectal surgeon. She also discussed the effect of her condition on her personal and family life, as well as the side effects of her treatment. Clinical presentation, pathogenesis, and management options were not reviewed, other than to advocate to the students that they should consider a biopsy when faced with a clinically evident lesion of uncertain diagnosis, as these topics had previously been discussed at length by the oral pathology faculty as part of the parent course in oral and maxillofacial pathology.

At University 1, all students in attendance $(\mathrm{N}=102)$ were given a paper questionnaire with seven open-ended questions in order to capture qualitative data. Questions focused on students' assessment of the messages acquired in the presentation, its effect on their future clinical decision making, and its overall effectiveness. An additional ten students participated in a focus group discussion led by one of the authors (K.E.F.). While there is no axiom for determining the sample size of a focus group, this number was chosen based on the view that six to ten people is a commonly accepted size. Interviews were transcribed, and the free text was analyzed to identify themes and subthemes in the narrative.

At University 2, all registered second-year students (104) were provided a link by email to a web-based survey four weeks after the PEP. The survey contained six questions consisting of both openended and ranking questions to capture qualitative data. Like the other survey, these questions focused on students' assessment of the messages acquired from the presentation, its effect on future clinical decision making, and its overall effectiveness. 
There were slight differences in wording between the two surveys, with a focus on open-ended comments in the survey administered at University 1. To allow for comparison between the two surveys, we assigned the responses to question \#1 at University 1 ("Do you believe the patient presenter's testimonial will affect your long-term memory [five years from now] about pemphigus vulgaris and mucous membrane pemphigoid?") to one of the following three response categories: "Yes, I feel very/somewhat strongly it will help me remember"; "I'm not sure if it will influence my future clinical decision making"; and "No, I don't think it will help me remember." Similarly, for University 2, the responses "I feel very strongly it will help me remember important facts about pemphigus" and "I feel somewhat strongly it will help me remember important facts about pemphigus" were combined into "Yes, I feel very/ somewhat strongly it will help me remember"; and the responses "It was interesting but I don't think it will help me remember important facts about pemphigus" and "It was not very useful and probably won't make a difference in what I remember about pemphigus" were grouped under "No, I don't think it will help me remember." The same consolidating approach was used for question \#2, "Will the patient presenter's testimonial about her illness experience change your future clinical decision making?"

For questions 3 ("What, if anything, did you find most effective about this presentation?") and 4 ("What, if anything, did you find least effective about this presentation?"), the open format nature of the questionnaire at both universities permitted a broad range of responses. Therefore, after reviewing all responses, we categorized them independently into the general themes.

Finally, all free-form responses were analyzed, and key themes were identified. A consensus conference was held by phone to agree on selection of these themes and tabulation of them. For quantitative questions, both median (the number separating the higher half of the data sample from the lower half) and interquartile ranges (statistical dispersion representing the difference between the upper and lower quartiles) were calculated.

\section{Results}

At University 1, 79 students (out of a possible 102; response rate $77.5 \%$ ) completed the surveys, and an additional ten students participated in the focus group. At University 2, 30 students (out of a possible 104; response rate $28.8 \%$ ) completed the survey.

The results showed that $94 \%$ and $100 \%$ (median $=5$, Interquartile Range $[\mathrm{IQR}]=1$ ) of respondents at University 1 and 2, respectively, reported the PEP would help their long-term recall of information about pemphigus. At University 1, $88 \%$ of the respondents to an open-ended question stated the PEP would influence their future clinical decision making (Table 1). This response mirrored the response to the similarly worded rating scale question at University $2(100 \%$; median $=5, \mathrm{IQR}=0)$.

Respondents at both universities (Table 2) commented that the most effective aspect of the PEP was the personal, emotional account of the patient's experience. The majority of comments also highlighted the fact that the presentation reinforced the importance of the doctor-patient relationship and the perception that a testimonial delivered in person is more relatable, memorable, and engaging than learning about it in a lecture. Additional comments related to how attendees would remember the painful nature of the patient educator's condition, the importance of not dismissing a patient's concerns, the need to develop a thorough differential diagnosis that does not overlook rare conditions, and the necessity of performing a biopsy to reach a definitive diagnosis. Comments also reflected on how the PEP emphasized the dentist's distinctive role in recognition, diagnosis, and possible management of these conditions.

General themes (Table 3) from the focus group discussion held at University 1 included a recommendation that the PEP include more clinical images of the condition, likely reflecting the visual learning preference of dental students and consistent with the written comments. All focus group participants reported having a solid understanding of the clinical presentation of pemphigus prior to the PEP. In addition, focus group participants stated that they were aware of the clinical presentation and major complications of these two conditions, as well as the protocols required to reach a diagnosis.

One of the most encouraging findings was the increased awareness among students of the importance of establishing a diagnosis before starting treatment, reflected by comments on the need for a biopsy prior to rendering a definitive diagnosis and on their recognition that not performing appropriate diagnostic testing can lead to a delay in diagnosis. Finally, the participants reflected that a personal testimonial "puts a face" to the condition, thereby reinforcing both the content taught in class and the importance 
Table 1. Students' assessment of patient educator presentation: comparison of percentages of total respondents at University $1(\mathrm{~N}=79)$ and University $2(\mathrm{~N}=30)$

1. Do you believe the patient presenter's testimonial will affect your long-term memory (5 years from now) about pemphigus vulgaris and mucous membrane pemphigoid?

Yes, I feel very/somewhat strongly it will help me remember

Unsure; it might help me remember

No, I don't think it will help me remember

Other response

No response

$\begin{array}{cc}94 \% & 100 \%^{\mathrm{a}} \\ 1 \% & 0 \\ 0 & 0 \\ 1 \% & 0 \\ 4 \% & 0\end{array}$

2. Will the patient presenter's testimonial about her illness change your future clinical decision making? Yes, I feel very/somewhat strongly it will influence my future clinical decision making

Unsure; I am not sure if it will influence my future clinical decision making

No, I feel somewhat/very strongly it will not influence my future clinical decision making

Other response

No response

$\begin{array}{cc}88 \% & 100 \%^{\mathrm{b}} \\ 2 \% & 0 \\ 3 \% & 0 \\ 3 \% & 0 \\ 4 \% & 0\end{array}$

3. What, if anything, did you find most effective about this presentation?

Personal account of her experience/emotions

$53 \%$

Openness/honesty/transparency/candidness/thoroughness

Good presentation skills

Will never misdiagnose this condition as a dentist (educational)

View of path to diagnosis from patient's point of view

Doctor-patient relationship

Other response

No response

$\begin{array}{cc}53 \% & 0 \\ 8 \% & 7 \% \\ 0 & 3 \% \\ 2 \% & 0 \\ 10 \% & 0 \\ 1 \% & 4 \% \\ 0 & 13 \%\end{array}$

4. What, if anything, did you find least effective about this presentation?

Nothing/NA

Extraneous details

Lack of images

Other response

No response

$\begin{array}{cc}47 \% & 50 \% \\ 0 & 3 \% \\ 22 \% & 0 \\ 0 & 0 \\ 31 \% & 47 \%\end{array}$

${ }^{a}$ Total response is combination of $72 \%$ very and $28 \%$ somewhat.

${ }^{\mathrm{b}}$ Total response is combination of $87 \%$ very and $13 \%$ somewhat.

of that content in the practice of dental medicine. When asked to compare different approaches to reinforcing didactic material, all 30 respondents at University 2 reported feeling that the in-person PEP was more effective than a videotaped presentation or written narrative. Moreover, a small number of students volunteered that they would like to see the PEP approach extended to other disease states.

\section{Discussion}

According to Lown et al., research on the benefits of patient involvement in the education of health care professionals has largely centered on patient assessment of interpersonal and communication skills, primarily based on standardized patient simulations. ${ }^{7}$ Substantially less has been written about the role of real patients in active teaching. Assessment of the published literature in this field is further complicated by the lack of consistent terminology employed to describe this type of patient involvement in health professions education. ${ }^{8}$ In their review of the relevant literature, Wykurz and Kelly identified only 23 articles with a primary focus on patients serving as active teachers involved in "facilitating learning and assessing the acquisition of knowledge, skills, and attitudes associated with medical practice." Furthermore, 13 of those studies had as their central focus an assessment of patient-directed teaching of physical examination skills, particularly as related to the musculoskeletal system.

Patient-driven participation is also used in the development and assessment of diagnostic skills, often by means of objective structured clinical examinations, although this approach is most ap- 
Table 2. Students' qualitative assessment of patient educator presentation: comparison of percentages of total respondents at University $1(\mathrm{~N}=79)$ and University $2(\mathrm{~N}=30)$

Importance of valuing the doctor-patient relationship (patient management)

\begin{tabular}{|c|c|}
\hline $100 \%$ & N/A \\
\hline $94 \%$ & $100 \%$ \\
\hline $89 \%$ & $100 \%$ \\
\hline $89 \%$ & $100 \%$ \\
\hline $78 \%$ & $\mathrm{~N} / \mathrm{A}$ \\
\hline $67 \%$ & $\mathrm{~N} / \mathrm{A}$ \\
\hline $65 \%$ & N/A \\
\hline $63 \%$ & $\mathrm{~N} / \mathrm{A}$ \\
\hline $62 \%$ & $73 \%$ \\
\hline $58 \%$ & $\mathrm{~N} / \mathrm{A}$ \\
\hline $43 \%$ & $\mathrm{~N} / \mathrm{A}$ \\
\hline $37 \%$ & $\mathrm{~N} / \mathrm{A}$ \\
\hline $33 \%$ & N/A \\
\hline $29 \%$ & $\mathrm{~N} / \mathrm{A}$ \\
\hline $28 \%$ & N/A \\
\hline $24 \%$ & $3 \%$ \\
\hline $23 \%$ & N/A \\
\hline $32 \%$ & N/A \\
\hline $22 \%$ & $7 \%$ \\
\hline $22 \%$ & N/A \\
\hline $16 \%$ & $\mathrm{~N} / \mathrm{A}$ \\
\hline $16 \%$ & $7 \%$ \\
\hline $13 \%$ & N/A \\
\hline $11 \%$ & $\mathrm{~N} / \mathrm{A}$ \\
\hline N/A & $27 \%$ \\
\hline N/A & $10 \%$ \\
\hline $10 \%$ & $60 \%$ \\
\hline $5 \%$ & $60 \%$ \\
\hline $4 \%$ & $\mathrm{~N} / \mathrm{A}$ \\
\hline $1 \%$ & $3 \%$ \\
\hline $\mathrm{N} / \mathrm{A}$ & $3 \%$ \\
\hline
\end{tabular}
Patient's personal testimony will affect long-term memory ( 5 years from now) about pemphigus (importance of topic)

Presentation will positively affect future dental practice (importance of topic)

Personal testimonial delivered in person is more effective, more memorable, more relatable, more engaging, and carries more impact than a typical lecture, textbook reading, and/or PowerPoint presentation; "puts a face to the disease" (importance of topic)

Biopsy required for diagnosis (patient management)

Including more clinical photos of lesion presentation during patient presentation, as an "aid in remembering this information" (importance of topic)

Valued the openness/honesty/candidness/thorough account from patient (best approaches to the patient presentation)

Importance of early recognition/diagnosis/treatment (importance of topic)

Personal account of presenter's experience and emotions (best approaches to the patient presentation)

Take action if lesions last for 2 weeks or longer (patient management)

Presentation resulted in better understanding/increased awareness and appreciation of the patient's experience and PV's personal impact (importance of topic)

Vigilance in approach to helping/treating future patients: asking questions, seeking answers, observing/responding, recognizing, and paying attention to details (patient management)

Being compassionate, empathetic, sensitive, caring, respectful, and professional (patient management)

Compelled to be more aware of symptoms and how to treat future patients with PV and/or similar diseases (importance of topic)

The view of the path to diagnosis from the patient's perspective (patient management)

Explore/consider all options and differentials and don't overlook rare conditions (patient management; importance of topic)

Recognize dentists' role in recognition/diagnosis/treatment (patient management)

CE courses, other similar patient presentations, and personal experience assessing PV patients as an "aid in remembering this information" (best approaches to the patient presentation)

Listen to patients/take symptoms and complaints seriously (patient management)

As a rare disease, there is a high probability of lack of exposure to the disease; as a "barrier to retaining the information from this presentation" (importance of topic)

Importance of specialist referral (patient management)

Good presentation skills; as a "most effective aspect of this presentation" (best approaches to the patient presentation)

Importance of putting on your "game face" (patient management)

The wealth of information learned and/or presented in dental school; as a "barrier to retaining the information from this presentation" (best approaches to the patient presentation)

Anger towards health professionals who failed to listen to her concerns or diagnose her condition; as a "most recollected detail of this presentation" (patient management)

Importance of thorough patient assessment to reach a definitive diagnosis; as a "most recollected detail of this presentation" (patient management)

Prolonged wait for definitive diagnosis; as a "most recollected detail of this presentation" (patient management)

Painful nature of condition and/or effect on her overall well-being; as a "most recollected detail of this presentation" (best approaches to the patient presentation)

Patient presentation reinforced oral pathology course information (best approaches to the patient presentation)

Compelled to never misdiagnose this condition as a dentist (patient management; importance of topic)

Extraneous details; as a "least effective/impactful aspect of this presentation" (best approaches to the patient presentation)

$\mathrm{PV}=$ pemphigus vulgaris 


\section{Table 3. Students' comments and themes from University 1 focus group $(\mathrm{N}=10)$}

More images (clinical photos of lesions throughout process of diagnosis and treatment) would help drive home the message/aid students in remembering/recognizing PV in the future.

Students had prior knowledge of PV and MMP prior to the patient presentation, including knowledge of the Nikolsky Sign; using direct immunofluorescence staining for biopsy; information about desquamative gingivitis; an understanding that cicatricial pemphigoid could affect the eyes; that PV is associated with intraepithelial separation; and that Pemphigoid is associated with sub-epithelial separation.

A personal testimony like the patient educator's will affect long-term memory (5 years from now) about pemphigus.

Students would use the following symptoms to help with their differential diagnosis of PV or MMP: bleeding gums, fluid ulcerations, blistering, and a positive Nikolsky Sign.

Prolonged wait for definitive diagnosis was the most recollected detail of the patient's testimony.

The patient presentation reinforced oral pathology course information.

CE courses, similar presentations to the patient's testimonial, and personal experience with PV patients would aid students in remembering this information.

Personal testimonial/experience delivered live/in-person is more effective, more memorable, more relatable, more engaging, and more impactful than a typical lecture, textbook reading, and/or PowerPoint slides. It makes the condition real and puts a face to the disease.

Biopsy.

Specialist referrals.

Good presentation skills were the most effective aspect of this presentation.

The wealth of information that is learned and/or presented in dental school is a barrier to retaining the information from the patient presenter's presentation.

Valued the openness/honesty/candidness/thorough account from patient speaker.

Presentation resulted in better understanding/increased awareness and appreciation of the patient's experience and PV's effect on the patient's life.

Be kind, compassionate, empathetic, sensitive, caring, respectful, and professional.

Important to recognize/diagnose/treat (early/quickly).

The fact that Pemphigus is a rare disease and there is a high probability of lack of exposure to it (even 20 years from now) is a barrier to retaining the information from the patient's presentation.

Importance of thorough patient assessment to reach a definitive diagnosis was the most recollected detail of patient's testimony.

$\mathrm{PV}=$ pemphigus vulgaris

plicable to one-on-one clinical education. Previous studies have suggested that patient-guided clinical examinations are complementary but not superior to physician educator-guided sessions - with patient educators providing a more systematic approach to teaching and more immediate and specific scripted feedback, whereas physician educators employ a more opportunistic approach, capitalizing on differences between clinical findings to highlight important or unusual features of the disease with a higher level focus on pathogenetic mechanisms. ${ }^{10,11}$ Nevertheless, students have reported reduced anxiety when developing their early clinical skills with patient educators, perceiving this approach as a nonthreatening learning environment. ${ }^{12}$

Benefits to the use of actual patients over simulated patients (actors who role-play a condition) include reduced intensity of training and increased satisfaction among the patient educators, as a common goal of theirs is to increase awareness of their disease process. Importantly, real patient educators appear to value their teaching role, regarding their involvement as making an important contribution to medical education "as experts and exemplars of their particular medical condition and as facilitators of the development of students' professional skills and attitudes." ${ }^{13}$ Likewise, health professions students and faculty members are, for the most part, supportive of some degree of involvement by patient instructors in the overall educational process, ${ }^{14}$ although some students have expressed concern that long-term reprise of a patient's story may result in decreased impact as the presentation becomes more rehearsed or "professionalized." 15 This potential would tend 
to be compounded by an obvious limitation to the PEP approach, especially where it requires one-onone patient-student interaction: the fact that only a small subset of patients with a particular disease are generally suited to take on this role.

However, one study found less support among faculty members and students for patients' taking a more prominent role in either curriculum development or formal student assessment since those responsibilities are typically assumed by faculty and, to a lesser extent, students. ${ }^{14}$ Studies of participation by real patients in active teaching have focused primarily on the role of the patient as a facilitator in developing students' professional skills and attitudes. Substantially less has been written concerning personal patient accounts of their journey to diagnosis and subsequent disease management, i.e., as exemplars of or spokespersons for their condition, especially with respect to lecture-based presentations. ${ }^{16}$ While a previous study in oral pathology-oral surgery considered patients' recollections of their personal experiences and interactions with members of the health care system, ${ }^{17}$ we found only one study that explored the potential benefits of this type of patient educator in dental education. ${ }^{18}$

Patient advocacy groups can potentially play an important role in this form of education by acting as sponsors of patient educators, although for the most part the focus of rare disease patient organizations has been on educating practicing health care professionals. ${ }^{19}$ The potential benefits of using patient educators during the training phase of a health care professional have been largely ignored.

A limitation of this study is that a direct comparison between the two student cohorts was complicated by the fact that the questionnaires were constructed differently. Nevertheless, the data support the hypothesis that the classroom presentation by a patient educator with a rare medical condition increased these students' understanding of the importance of learning about rare conditions that can present with oral manifestations and emphasized the importance of rare disease knowledge retention through an emotional appeal. Although this study did not collect long-term data to support the expectation that the PEP will increase students' retention of information, we suggest that the PEP increased students' awareness that their patient's presentation might be related to any number of potential rare conditions. Furthermore, one study found evidence to suggest that, in the context of problem-based learning, using real patients to present their symptoms (in that case, actors) as opposed to paper scenarios can be a potent stimulus to encourage health professions students to more fully research the disease process. ${ }^{20}$

A number of participants in our study commented that they would like to see presentations from additional patients with other conditions. However, it is not clear if doing so would offer any educational advantage. It can be argued that, in the case of the patient presenter format used in this study, in which a rare condition is presented, the educational objective is to increase student awareness of the need to be fully cognizant of the full range of conditions, both common and rare, explored during training, both to increase the likelihood of reaching a correct diagnosis and to foster empathy towards a patient's condition. In the case of rare diseases, such as pemphigus vulgaris, greater understanding of the actual disease process can arguably be viewed as a secondary end point to the PEP. This result is in potential conflict with patient educators' motivation of advocating for increased awareness of their particular condition. However, the broader view should be taken, namely, that this represents a mutually beneficial relationship as increased awareness of the possibility of encountering these rare conditions among health care professionals would be expected to increase their likelihood of including such conditions in their differential diagnosis and hence pursuing a more thorough diagnostic assessment for their patients.

Prior to introducing PEPs into the dental curriculum, certain considerations must be anticipated. First and foremost, the patient should have a compelling narrative to offer, ideally with three or four take-home messages (in our patient lecture, the points were that dentists play a critical role in early diagnosis; a patient's concerns should not be ignored; although some conditions are extremely rare, the possibility that your patient presents with one of them cannot be ignored; and if a lesion of uncertain etiology is identified, a biopsy is needed, not just to rule out cancer but to arrive at a definitive diagnosis so that proper treatment can be begun). Any patient who volunteers to present an overview of his or her condition needs to have a solid understanding of the pathogenesis of the disease process. A reasonable level of communication skills and teaching ability is obviously also very important if the lecture is to be at all memorable. For patients who do not come with recommendations from other educators, it would be advisable to have a dry run of the presentation prior 
to the actual session, so that effectiveness, content, and suitability for the audience can be confirmed. The patient educator should be amenable to modifying the presentation, based on feedback, from either student attendees or the faculty organizer. A 40- to 45-minute block of time, with additional time for student questions, is recommended. Also, the benefits of using this time for a patient presentation must justify this use of class time.

In the scenario in our study (a patient with a rare disease with an incidence of 1:500,000-1:1,000,000), identifying patient educators willing to present their story to a large group of students is challenging. Potential presenters may be identified through a practitioner's own group of patients or through a patient advocacy group. The former is potentially awkward, as directly recruiting patients of record is fraught with ethical concerns: is the patient agreeing to act as a presenter because of genuine interest in doing so, or could the patient subconsciously experience some degree of unintended coercion to agree to his or her provider's request? Realistically, to avoid even the semblance of an ethical quandary, a patient would ideally be the one to broach the idea of wanting to help promote awareness of the condition.

A plan for sustainability should also be developed, in that health professions educators must be able to continuously identify new potential patient presenters since current ones may move, decide they are no longer interested in presenting their story, or undergo other life changes. Again, the selection of patients with a particular condition may be less important than identifying potential patient educators who have a clear message to offer. Finally, as noted by the participants, inclusion of high-quality pretreatment and posttreatment clinical images of the condition would reinforce the visual learning of dental students, increasing the impact of the PEP and helping to associate the appearance of lesions with the story of the patient's disease.

While this approach was initially presented and evaluated as a way to enhance knowledge of pemphigus, it was clear from the participants' feedback that this approach increased awareness of and interest in learning about less common conditions in general. At the formative stage of dental students' education, this approach carries significant potential benefit at a rather modest investment since a single patient educator can impact the professional development of an entire dental class in a single one-hour presentation.

\section{Conclusion}

The potential benefits of a presentation by a real patient on his or her own illness have rarely been discussed in the dental education literature. In our mixed-methods study, comprising both quantitative and qualitative analysis, we found that a classroom presentation by a patient educator with a rare medical condition increased the dental students' self-reported recognition of the importance of learning about rare conditions with oral manifestations and emphasized the importance of rare disease knowledge retention. Future research should assess the long-term effect of these types of patient educator interventions on the primary outcome of learner behavior.

\section{Acknowledgments}

We wish to thank our patient educator, who bravely shares her story with hundreds of dental students across the United States. We wish to thank the Indiana University Foundation Oral Pathology Fund, the Sy Syms Foundation, and The Unger Family for their generous financial support of this project. We also thank Dr. Carol Anne Murdoch-Kinch for referring our patient educator to us and Dr. Susan Zunt for recommending Dr. Krutchkoff and Eisenberg's article "Dying of cancer: a patient's recollection of her illness and of her doctors," Oral Surg Oral Med Oral Pathol 1991;71:401-6.

\section{Disclosure}

Paul Edwards is a member of the Dental Advisory Council and the Awareness Campaign Committee of the International Pemphigus \& Pemphigoid Foundation (IPPF). The IPPF is a global organization dedicated to improving the quality of life of all people diagnosed with, or affected by, pemphigus or pemphigoid. Its awareness campaign, www.pemphigus. org/awareness, aims to reduce diagnostic delays of pemphigus and pemphigoid patients. Based on data from this study, the organization has committed to sponsoring patient educator presentations at a minimum of 19 selected dental schools across the United States.

\section{REFERENCES}

1. Olowo-Ofayoku A, Moxham BJ. Comparisons between the attitudes of medical and dental students toward the clinical importance of gross anatomy and physiology. Clin Anat 2014;27:976-87. 
2. Spencer J, Blackmore D, Heard S, et al. Patient-oriented learning: a review of the role of the patient in the education of medical students. Med Educ 2000;34:851-7.

3. National Institutes of Health, Genetic and Rare Disease Information Center. Rare diseases. At: rarediseases.info. nih.gov. Accessed 25 Mar. 2016.

4. Edwards PC. "Considering that this is such a rare condition, should we really be expected to recognize it?" Oral Surg Oral Med Oral Pathol Oral Radiol Endod 2011;112:143-5.

5. Murphy RJ, Gray SA, Straja SR, Bogert MC. Student learning preferences and teaching implications. J Dent Educ 2014;68(8):859-66.

6. Finch H, Lewis J. Focus groups. In: Ritchie J, Lewis J, eds. Qualitative research practice: a guide for social science students and researchers. London: Sage, 2005:170-98.

7. Lown BA, Sasson JP, Hinrichs P. Patients as partners in radiology education: an innovative approach to teaching and assessing patient-centered communication. Acad Radiol 2008;15:425-32.

8. Towle A, Bainbridge L, Godolphin W, et al. Active patient involvement in the education of health professionals. Med Educ 2010;44:64-74.

9. Wykurz G, Kelly D. Developing the role of patients as teachers: literature review. BMJ 2002;325:818-21.

10. Oswald AE, Wiseman J, Bell MJ, Snell L. Musculoskeletal examination teaching by patients versus physicians: how are they different? Neither better nor worse, but complementary. Med Teach 2011;33:227-35.

11. Hendry GD, Schrieber L, Bryce D. Patients teach students: partners in arthritis education. Med Educ 1999;33:674-7.
12. Jha V, Setna Z, Al-Hity A, et al. Patient involvement in teaching and assessing intimate examination skills: a systematic review. Med Educ 2010;44:347-57.

13. Bokken L, Rethans JJ, Scherpbier AJ, van der Vleuten CP. Strengths and weaknesses of simulated and real patients in the teaching of skills to medical students: a review. Sim Healthcare 2008;3:161-9.

14. Jha V, Quinton ND, Bekker HL, Roberts TE. What educators and students really think about using patients as teachers in medical education: a qualitative study. Med Educ 2009;43:449-56.

15. Jha V, Quinton ND, Bekker HL, Roberts TE. Strategies and interventions for involvement of real patients in medical education: a systematic review. Med Educ 2009;43: 10-20.

16. Stacy R, Spencer J. Patients as teachers: a qualitative study of patients' views on their role in a community-based undergraduate project. Med Educ 1999;33:688-94.

17. Krutchkoff DJ, Eisenberg E. Dying of cancer: a patient's recollection of her illness and of her doctors. Oral Surg Oral Med Oral Pathol 1991;71:401-6.

18. Renard E, Alliot-Licht B, Gross O, et al. Study of the impact of patient-educators on the course of basic sciences in dental studies. Eur J Dent Educ 2015;19:31-7.

19. Gizycki V. Contribution of rare disease patient organizations to medical education. Orphanet J Rare Dis 2010;5(Suppl 1):O25.

20. Dammers J, Spencer J, Thomas M. Using real patients in problem-based learning: students' comments on the value of using real, as opposed to paper cases, in a problembased learning module in general practice. Med Educ 2001;35:27-34. 